\title{
Measurement for the Thickness of Water Droplets/Film on a Curved Surface with Digital Image Projection (DIP) Technique
}

\author{
Lingwei Zeng ${ }^{1}$, Hanfeng Wang ${ }^{1,2}\left(\mathbb{D}\right.$, Ying $\mathrm{Li}^{1,3, *}$ and Xuhui $\mathrm{He}^{1,2} \mathbb{D}$ \\ 1 School of Civil Engineering, Central South University, Changsha 410075, Hunan, China; \\ 174812223@csu.edu.cn (L.Z.); wanghf@csu.edu.cn (H.W.); xuhuihe@csu.edu.cn (X.H.) \\ 2 National Engineering Laboratory for High-speed Railway Construction, Central South University, \\ Changsha 410075, Hunan, China \\ 3 Department of Mechanical Engineering, The Hong Kong Polytechnic University, Hung Hom, Kowloon, \\ HongKong Special Administrative Region 999077, China \\ * Correspondence: carlos.li@connect.polyu.hk
}

Received: 19 February 2020; Accepted: 22 April 2020; Published: 24 April 2020

check for updates

\begin{abstract}
Digital image projection (DIP) with traditional vertical calibration cannot be used for measuring the water droplets/film on a curved surface, because significant systematic error will be introduced. An improved DIP technique with normal calibration is proposed in the present paper, including the principles, operation procedures and analysis of systematic errors, which was successfully applied to measuring the water droplets/film on a curved surface. By comparing the results of laser profiler, traditional DIP, improved DIP and theoretical analysis, advantages of the present improved DIP technique are highlighted.
\end{abstract}

Keywords: water droplets/film; curved surface; digital image projection DIP; image processing technique

\section{Introduction}

The properties of water droplets/film on a surface are of great significance in the aviation engineering, civil engineering, thermal engineering, etc. Icing is widely considered as one of the most serious weather hazards to aircraft. When an aircraft flies through clouds or fog containing super-cooled small water droplets, icing will occur after those droplets impact on the airfoil (Mason [1]). Under incompletely freezing conditions, only a fraction of the water freezes in the impact area. Other water runs back, driven by the boundary flow over the surface. This phenomenon results in the change of water distribution on the airfoil, forming glaze ice outside the impact area (Politovich [2], Hu and Huang [3] and $\mathrm{Hu}$ and Jin [4]). The shape of glaze ice is complicated and hard to predict accurately, which may significantly deteriorate the aerodynamic performance of airfoil.

Stay cables of bridge are prone to large amplitude vibrations especially when rain and wind occur simultaneously, which is called rain-wind-induced vibration (RWIV). The upper rivulet has significant influences on the RWIV of the cable since its circumferential movement shifts the flow around the cable from subcritical to supercritical regions alternately (Seidel and Dinkler [5]). Quantitative information of the water rivulets, including thickness, width and position, is essential to reveal the mechanisms of RWIV (Flamand [6] and Du et al. [7]).

The measurement of rivulets is also important in the thermal engineering. When a rivulet flows on a solid surface, it shares an interface with the surrounding gas. The rivulet is crucial for investigating heat exchange and gas absorption process (Rekstad et al. [8]). Moreover, it is also indispensable to obtain the precise information of the liquid film flow on nuclear fuel rods when analyzing the critical heat flux under postulated loss-of-coolant accidents in boiling water reactors (Hoshiide et al. [9] and Yano et al. [10]). 
Recently, Hu et al. [11] and Zhang et al. [12] proposed a digital image projection (DIP) technique, which was successfully used to measure the instantaneous pattern of water rivulets and film on a NACA0012 aerofoil with the chord length of $101 \mathrm{~mm}$.

The principle of the DIP technique is briefly introduced for readers here. As shown in Figure 1, when there is no droplet on the plane, a projected image from the projector (point $\mathrm{N}$ ) falls on point $\mathrm{A}$. If an opaque droplet appears on the plane, the light will fall on its surface at point $\mathrm{D}$. However, from the view of the camera (point $\mathrm{M}$ ), the image moves to point $\mathrm{C}$. The distance between $\mathrm{A}$ and $\mathrm{C}$, i.e., $\overline{A C}$, can be determined based on cross-correlation between the two projected images taken with and without the droplet.

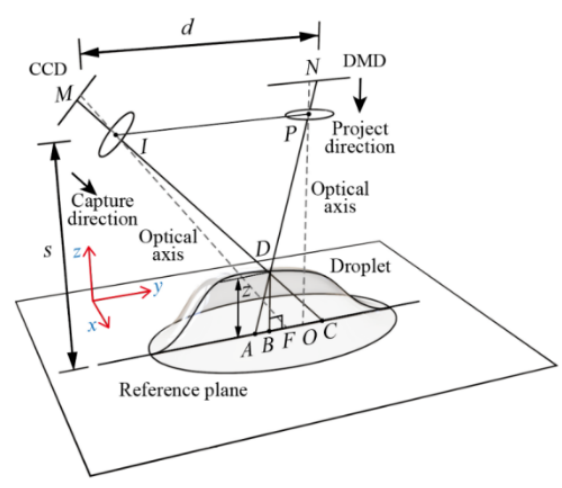

Figure 1. Schematic diagram of the digital image projection (DIP) technique (Zhang et al. [12]).

Assume that the horizontal distance between the projector and the camera is $d$, the reference plane is parallel to them with a distance $s$. From the relationship between $\triangle M N D$ and $\triangle A C D$, the following relation applies:

$$
\frac{d}{\overline{A C}}=\frac{s-\overline{B D}}{\overline{B D}} \approx \frac{s}{\overline{B D}}
$$

(the distance $s$ is usually much larger than $\overline{B D}$ ).

According to this relation, the thickness of the droplet, $z(x, y)$, can be obtained:

$$
z(x, y)=\overline{B D} \approx \frac{s}{d} \overline{A C}=k_{1} \overline{A C}
$$

The conversion coefficient $k_{1}$, i.e., the displacement-to-height conversion constant, can be determined through a so-called "vertical calibration procedure" for a given DIP system. By utilizing vertical calibration procedure, the coefficient $k_{1}$ in Equation (2) can be obtained.

However, the traditional DIP method assumes the area, on which the water rivulet is measured, is flat or of exceedingly small curvature (e.g., an aerofoil). Thus, the calibration is only conducted in the direction perpendicular to the plane (Hu et al. [11] and Zhang et al. [12]). Consequently, the traditional DIP technique cannot be applied directly to a surface with large curvature. Otherwise, significant systematic error will be introduced. The present paper proposes a new calibration method to overcome this issue, which allows the DIP technique to be applied to curved surface.

\section{Technical Principles of the Improved DIP Method}

\subsection{Methodology}

For the measurement on a curved surface (as shown in Figure 2a), if the preceding vertical calibration is still adopted, the measured thickness $\overline{D B}$ will be in the vertical direction. However, the real thickness of the droplet is $\overline{D H}$, which is normal to the surface. Obviously, as illustrated in Figure $2 b$, the systematic bias between $\overline{D B}$ and $\overline{D H}$ depends on the included angle $\alpha$ between them. With the increase of $\alpha$, the systematic bias raises quickly. Moreover, the ratio of the real thickness $d(\overline{D H})$ and the 
local radius of the curved surface $r$ also contributes to the systematic bias. This effect will be magnified when $\alpha$ becomes larger. That is, the traditional DIP overestimates the thickness of droplets on a curved surface. If the accepted error level is $5 \%$, the traditional DIP technique must be improved to eliminate this systematic error when $\alpha$ is larger than $17^{\circ}$, even when $d / r$ approaches zero.

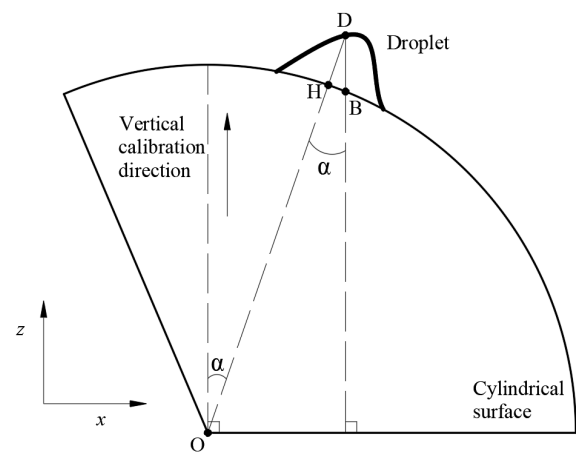

(a)

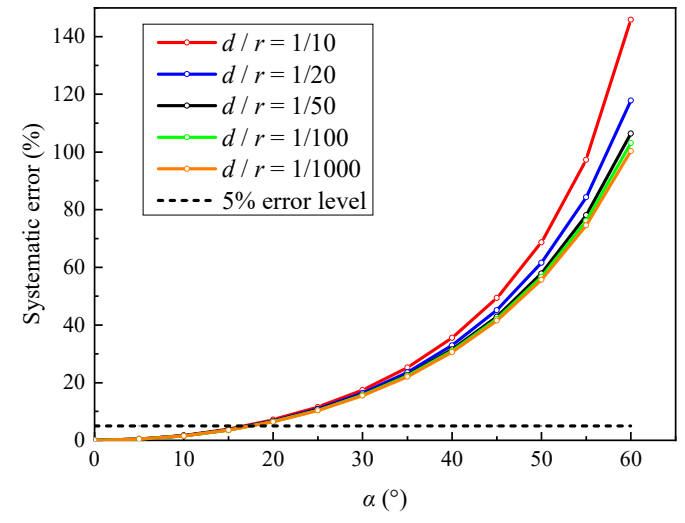

(b)

Figure 2. Schematic diagram of (a) a droplet on the cylindrical surface and (b) dependence of the systematic error on $\alpha$ for different $d / r$.

Consequently, a correction coefficient $k_{2}\left(=\frac{\overline{D H}}{\overline{D B}}\right)$ is necessary to eliminate the above-mentioned bias for applying the DIP technique on a curved surface. Figure 3a shows a typical geometric configuration of the DIP measurement on a curved surface. The projected light falls on an arbitrary curved surface at point $\mathrm{A}$ with an angle $\theta$. The angle between radius $\overline{O A}$ and the vertical direction, $\overline{O G}$, is $\alpha$. It should be mentioned that $\alpha$ can be negative when the projected light falls on the left side of $\overline{O G}$.

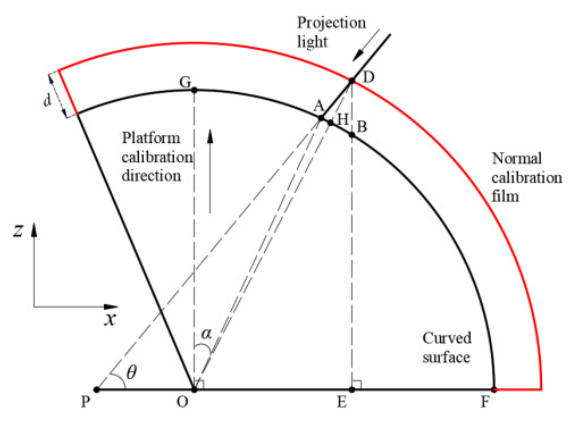

(a)

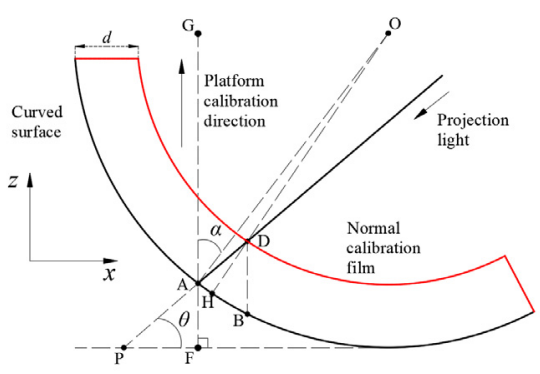

(c)

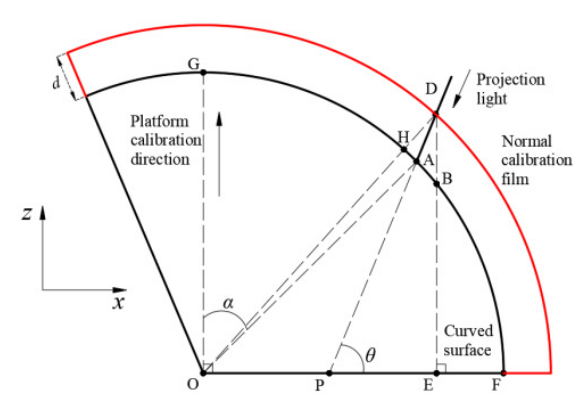

(b)

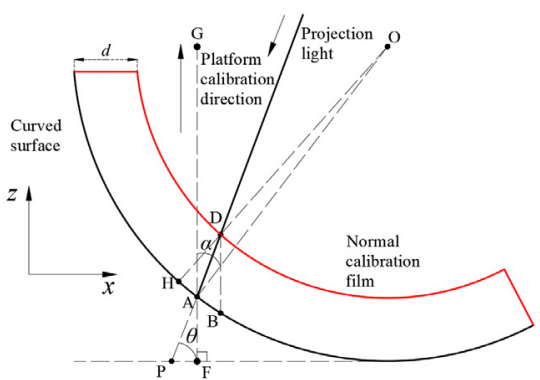

(d)

Figure 3. Typical geometric configurations of the DIP measurement on curved surface: (a) $90^{\circ}-\alpha \geq$ $\theta$ (convex surface); (b) $90^{\circ}-\alpha \leq \theta$ (convex surface); (c) $90^{\circ}-\alpha \geq \theta$ (concave surface); (d) $90^{\circ}-\alpha \leq \theta$ (concave surface) 
If the original curved surface expands to its new position with a normal displacement of $d(\overline{D H})$, as shown in Figure 3a. Assume that the radius of the curved surface $r$ is much larger than $d$. Under the condition of $90^{\circ}-\alpha \geq \theta$, the thickness $\overline{B D}$, acquired by the previous vertical calibration procedure, can be calculated by Equations (3)-(5):

$$
\begin{gathered}
\overline{B D}=(r+d) \cdot \cos \angle O D E-\overline{B E} \\
\overline{B E}=\sqrt{r^{2}-[(r+d) \cdot \sin \angle O D E]^{2}} \\
\angle O D E=\angle A D E-\angle A D H=90^{\circ}-\theta-\angle A D H
\end{gathered}
$$

According to the law of sines:

$$
\frac{\overline{O A}}{\sin (\angle A D H)}=\frac{\overline{D H}+\overline{H O}}{\sin (\angle O A D)}
$$

Because $\angle O A P+\angle O A D=180^{\circ}$ and $\angle O A P=90^{\circ}-\alpha-\theta$, thus:

$$
\sin (\angle O A D)=\sin (\angle O A P)=\sin \left(90^{\circ}-\alpha-\theta\right)
$$

Hence, Equation (6) can be simplified according to Equation (7) as follows:

$$
\angle A D H=\sin ^{-1}\left[\frac{r \cdot \sin \left(90^{\circ}-\alpha-\theta\right)}{r+d}\right]
$$

Based on the discussion above, in a certain interrogation window on the surface (i.e., $r$, $\alpha$ and $\theta$ are constant), the thickness, $\overline{B D}$, is only determined by the normal displacement, $d$. Meanwhile, the correction coefficient $k_{2}\left(=\frac{d}{\overline{B D}}\right)$ can be also determined.

Figure $3 b-d$ present the other three possible configurations for the application of the DIP measurement on a curved surface. Based on similar analysis procedure, $k_{2}$ for these configurations can all be determined.

Figure 4 illustrates the relationship between $d^{*}$ (normalized with the local radius of the curved surface $r$ ) and $k_{2}$, calculated from Equations (3)-(8). $k_{2}$ decreases gradually with the increasing $d^{*}$. Obviously, $k_{2}$ and $d^{*}$ show good linear relationship when $d^{*}$ ranging from 0 to 0.1 .
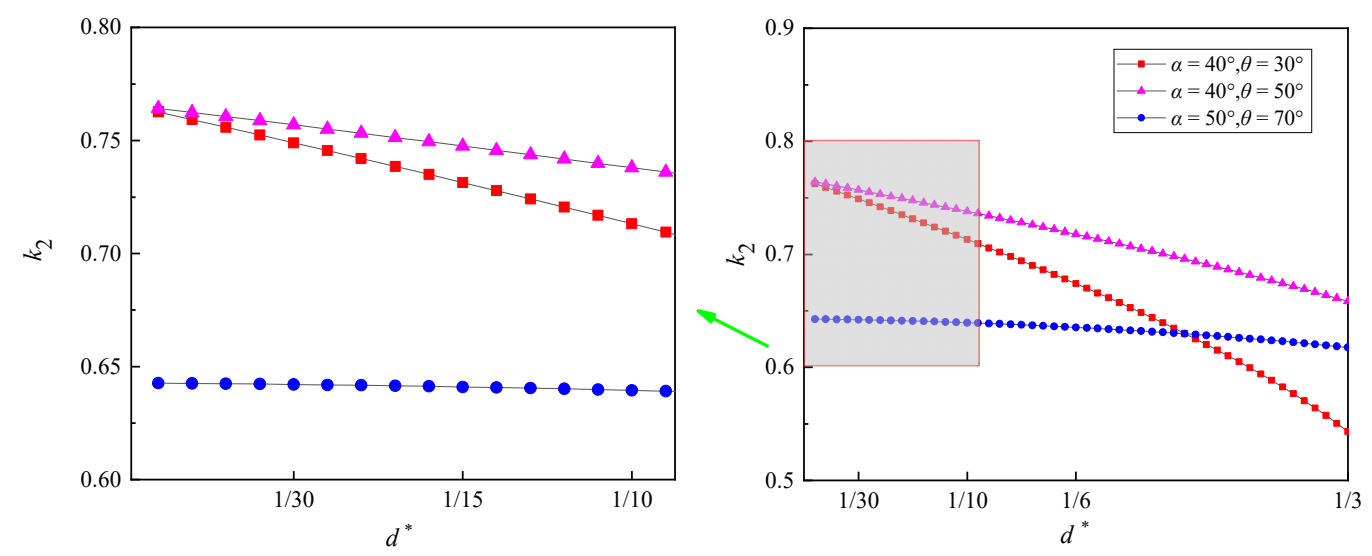

Figure 4. Relationship between $d^{*}$ and the corrected coefficient $k_{2}$.

Assume that the linear relation between $d^{*}$ and $k_{2}$ is:

$$
k_{2}=\frac{d^{*}}{\overline{B D}^{*}}=\frac{d}{\overline{B D}}=C_{1} \cdot d^{*}+C_{2}=C_{3} \cdot d+C_{4}
$$


where $\overline{B D}^{*}$ is normalized with the local radius of the curved surface $r$.

According to Equation (2), Equation (9) can be expressed as:

$$
\frac{d}{\overline{A C}}=k_{1} \cdot C_{3} \cdot d+k_{1} \cdot C_{4}
$$

In a certain interrogation window on the surface, $k_{1}$ is a constant (Zhang et al. [12]). Hence, Equation (10) indicates that $\frac{d}{\overline{A C}}$ and $d$ are also quasi-linearly correlated. Thus, it is acceptable to get the $\frac{d}{\overline{A C}}-d$ relation by measuring $\overline{A C}$ at only two specific $d$.

If we can attach two shells with given thickness to the curved surface, respectively, then the linear relation between $\frac{d}{A C}$ and $d$ can be acquired. This calibration is named for "normal calibration" hereafter in the present paper. It can be obtained:

$$
d=\frac{-C_{4} \cdot k_{1} \cdot \overline{A C}}{C_{3} \cdot k_{1} \cdot \overline{A C}-1}
$$

In Equation (11), the distance $\overline{A C}$ between the two images can be determined based on cross-correlation between the tested images and reference image. The constant coefficients $k_{1} \cdot C_{3}$ and $k_{1} \cdot C_{4}$ can be calculated by normal calibration procedure. Hence, the normal displacement, $d$, can be also computed by Equation (11).

\subsection{Error Analysis}

The $k_{2}-d^{*}$ in normal calibration is assumed to be perfectly linear. The systematic error caused by this assumption is related to $\alpha, \theta$ and the ratio of $d_{\max }$ (the maximum normal displacement) and $r$. By optimizing the setup arrangement, e.g., $\alpha$ and $\theta$, this systematic error can be controlled within an acceptable level. Figure 5 shows the dependence of this systematic error on the two main influencing factors $\alpha$ and $\theta$ for different $r$ and $d_{\max }$. The thickness of two hypothetical shells for normal calibration is set as $\frac{1}{3} d_{\max }$ and $\frac{2}{3} d_{\max }$.

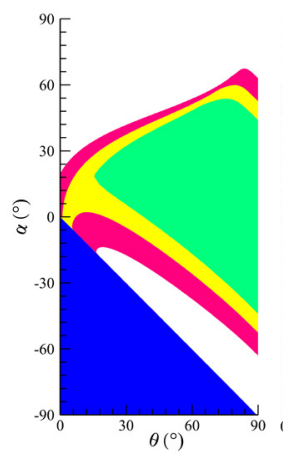

(a)

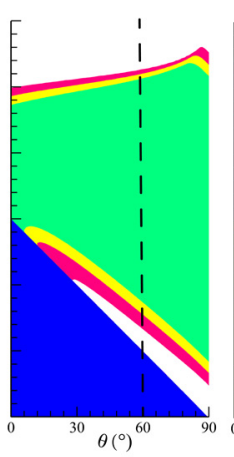

(b)

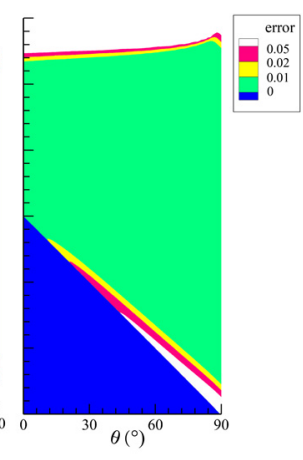

(c)

Figure 5. Maximum systematic error with different combinations of $\theta$ and $\alpha$ : (a) $d_{\max } / r=1 / 3$; (b) $d_{\max } / r=1 / 10 ;$ (c) $d_{\max } / r=1 / 33$.

For better illustration, Figure 6 displays the systematic error under the condition of $d_{\max } / r=\frac{1}{10}, \theta=60^{\circ}$, marked with the black dashed line in Figure $5 \mathrm{~b}$. Note that, as shown in Figure 3, $\theta$ is determined by the position and the incidence angle of the projector and $\alpha$ represents the location of the interrogation window to be tested. As expected, both $\alpha$ and $\theta$ have obvious effects on the systematic error. In Figure 6, the blue region on the left side of OA is out of the field illuminated by the projected light. Based on the information provided by Figures 5 and 6 and Equation (1), the projector should be mounted far away from the reference surface. Meanwhile, in order to obtain a larger green area, the recommended angle $\theta$ is about $80^{\circ}$. Moreover, rather than the standard reference axis (vertical 
direction and horizontal direction) reported by Zhang et al. [12], as shown in Figure 6a, the reference axis can be adjusted arbitrarily, making the tested region within the green area in order to obtain precise results.

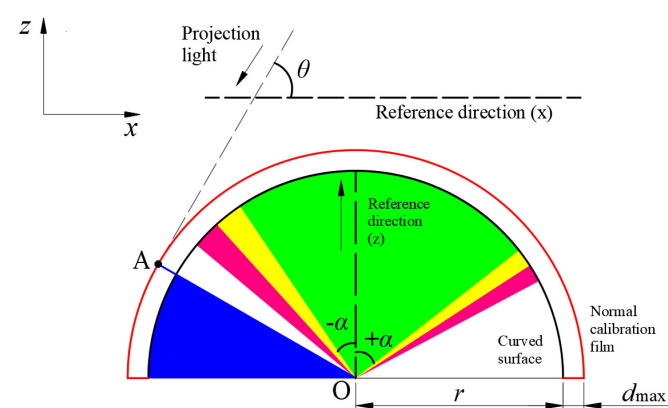

(a)

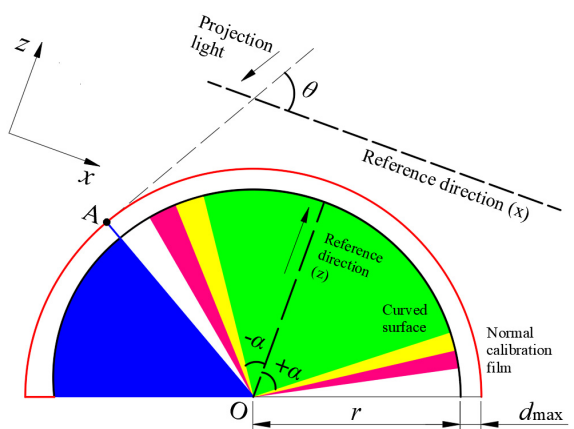

(b)

Figure 6. Systematic error under the condition of $d_{\max } / r=\frac{1}{10}, \theta=60^{\circ}$ utilizing (a) standard reference axis or (b) arbitrary reference axis.

Likewise, the ratio of $d_{\max }$ and $r$ also has remarkable influences on the systematic error. According to Figure 5, the systematic error reduces with the decrease of the ratio of $d_{\max }$ and $r$ and vice versa.

\subsection{Experimental Setup}

A grayscale image consists of particles with random gray values generated by Matlab was used as the projected image, as shown in Figure 7. The gray level of this projected image should be adjusted according to the ambient lighting condition. Because the combined effects of intense ambient lighting condition and high gray level may lead to the improper focusing of the camera. Thus, it is recommended that the experiments are conducted in a dark environment. Meanwhile, the gray level of the projected image should not be too high.

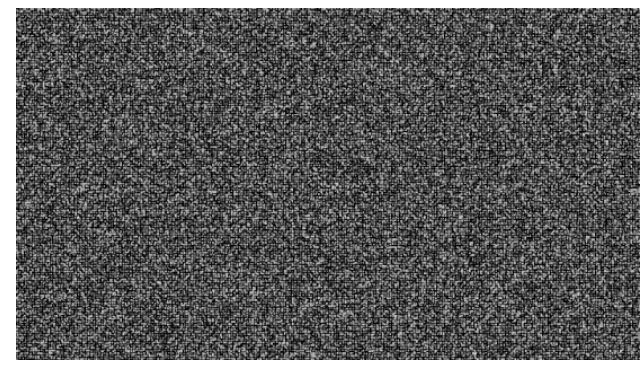

(a)

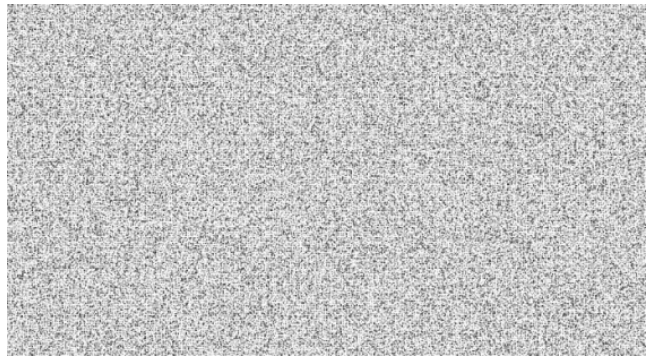

(b)

Figure 7. Projected image with random scattered particles: (a) low grayscale; (b) high grayscale.

In the present experiment, a digital projector (Robot go 3D, produced by Aiteqi Technology Company Limited, Shenzhen, China; the resolution was $1280 \times 800 \mathrm{pixel}^{2}$ ) was used to cast the random scatter particle image onto a reference surface for the DIP measurement. And a digital single-lens reflex (DSLR) camera (Canon EOS 6D, produced by Canon Company Limited, Tokyo, Japan; the highest resolution was $5472 \times 3648$ pixel $^{2}$, the valid pixel was 20 million) was applied for capturing images. The measured surface was one-third of a cylindrical surface with the length of $70 \mathrm{~mm}$ and radius of $50 \mathrm{~mm}$. It was produced by a rapid 3D prototyping machine using white resin material and carefully polished. Two normal calibration shells, with the thickness of $1 \mathrm{~mm}$ and $2 \mathrm{~mm}$, respectively, were prepared.

A high-resolution 2D Laser Profiler (Keyence LJ-G200, produced by Keyence Corporation, Itasca, IL, USA; the length of the linear laser was $62 \mathrm{~mm}$, the accuracy was up to $2 \mu \mathrm{m}$ ) was also used to measure the thickness of the droplet, which provided the validation for the present DIP technique. 
The normal calibration procedure can be divided into the following steps:

1. Based on the measurement region, fix the projector and camera according to the result shown in Figures 5 and 6.

2. Project the image onto the tested curved surface. Take a picture of the projected image without water droplet or rivulet, which will be used as "original image".

3. Attach the two thin shells with given thicknesses to the measured surface, respectively. Take pictures of the projected image on each of the two shells. These two pictures will be used as "normal calibration images".

4. Remove the thin shells. Make droplets or rivulets attached on the surface. Then, the projected image will be distorted with the appearance of these water droplets or rivulets. Capture the distorted images, which contain the geometric information of the water droplets or rivulets.

5. Calculate the constant coefficients $k_{1} \cdot C_{3}$ and $k_{1} \cdot C_{4}$ with cross-correlation between the "normal calibration images" and "original image".

6. Calculate the thickness of water droplets or rivulets with cross-correlation between the distorted images and "original image"

Noted that, in order to enhance the contrast of the projected image on the free surface of the water droplet, a very small amount of titanium pigment should be added into the water.

\subsection{Interrogation Window Size}

The interrogation window (IW) size does influence the measurement results. For the present experiment, the resolution of the camera was $5472 \times 3648$ pixel $^{2}$ (which was not completely used for cross-correlation, only the part containing the droplet was adopted for the measurement), each pixel indicates $0.05 \mathrm{~mm}$. The cross-correlation algorithm provides one result for each IW according to the image displacement within it. It was similar to a spatial filtering or average for each IW. Thus, a larger IW reduces the spatial resolution of the measurement. However, if the IW is set to small, the cross-correlation algorithm may not provide a reliable result, because the image within each IW is too small to provide enough information for cross-correlation. The smallest applicable IW largely depends on the quality of the image captured.

For the present experiment, the effects of IW on the results were tested during calibration process. Since the thickness of the calibration shell was given and uniform, the cross-correlation was conducted for different IWs and their corresponding RMS values were calculated, as shown in Figure 8. As expected, the Root Mean Square (RMS) value reduces quickly with increasing the size of IW and becomes stable for the IW larger than $18 \times 18$ pixel $^{2}\left(0.9 \times 0.9 \mathrm{~mm}^{2}\right)$. Consequently, the IW of $18 \times 18$ pixel $^{2}$ was utilized in the experiment.

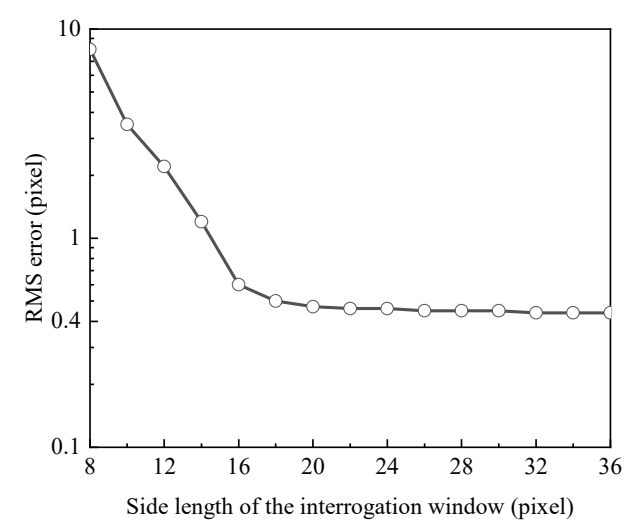

Figure 8. Root Mean Square (RMS) error as function of the interrogation window size. 


\section{Applications}

\subsection{Measurement for a Curved Uniform Shell}

The present DIP technique was utilized to measure a curved uniform thin shell with a given thickness of $1.5 \mathrm{~mm}$ to test its applicability. The reference surface was one-third of a cylindrical surface with the length of $70 \mathrm{~mm}$ and radius of $50 \mathrm{~mm}$ (the same with that introduced in Section 2.3), as shown in Figure 9a. The tested thin curved shell was attached firmly on the reference surface. Figure $9 \mathrm{~b}$ shows the results along the central cross-section (marked with a red dashed line in Figure 8a obtained by the DIP technique with the traditional vertical calibration and the present normal calibration, respectively. The result was normalized with the given thickness of the tested shell $(1.5 \mathrm{~mm})$. Obviously, the thickness obtained by the traditional DIP method increases with the absolute value of $\alpha$, while the present normal calibration technique provides more accurate results within $\alpha$ from $-35^{\circ}$ to $35^{\circ}$.

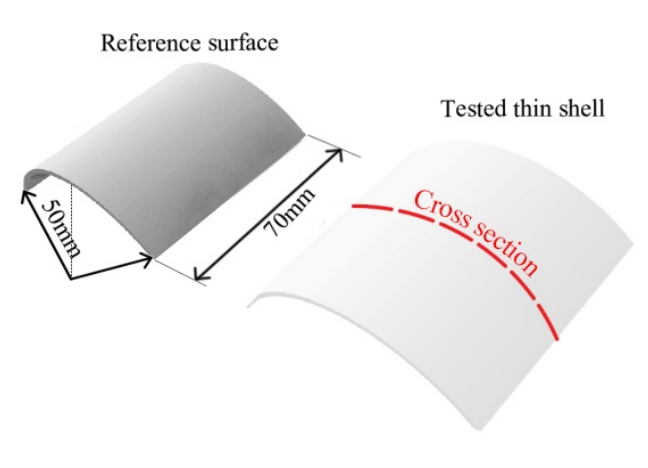

(a)

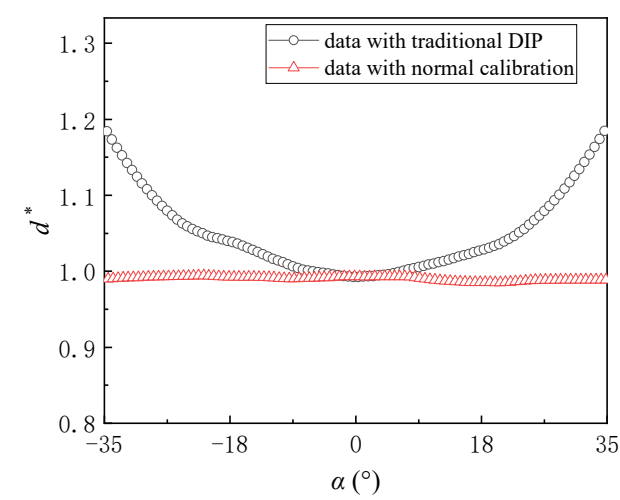

(b)

Figure 9. Measurement for a curved shell: (a) experimental models; (b) results along the central cross section.

\subsection{Measurement for the Water Droplet on a Flat Surface}

A high-resolution 2D Laser Profiler (Keyence LJ-G200, produced by Keyence Corporation, Itasca, IL, USA;) was used to measure the thickness of a droplet to validate the present DIP method. The measurements were conducted on a flat and a cylindrical surface, respectively. The Laser Profiler was mounted on a 2D traverse system with an accuracy of $0.02 \mathrm{~mm}$, which was used to move the Laser Profiler to scan the droplet, as shown in Figure 10a. Figure 10b,c present the measurement results of the droplet on a flat surface using the Laser Profiler and DIP technique, respectively. Figure 10d,e qualitatively compare the thickness along the two typical lines (Line 1 and Line 2 in Figure 10b,c, which cross the apex of the droplet. The measurement results using the above mentioned two techniques accord quite well with each other, except for the slight distortion of the DIP result along Line 2. The distortion in the horizontal direction was caused by the slant arrangement of the camera in that direction, as shown in Figure 10a. This distortion of DIP technique can be neglected when applying to large area measurement (Hu et al. [11] and Zhang et al. [12]). Apparently, it can be confirmed that the DIP technique is applicable for measuring the droplet/rivulet on a flat surface. 


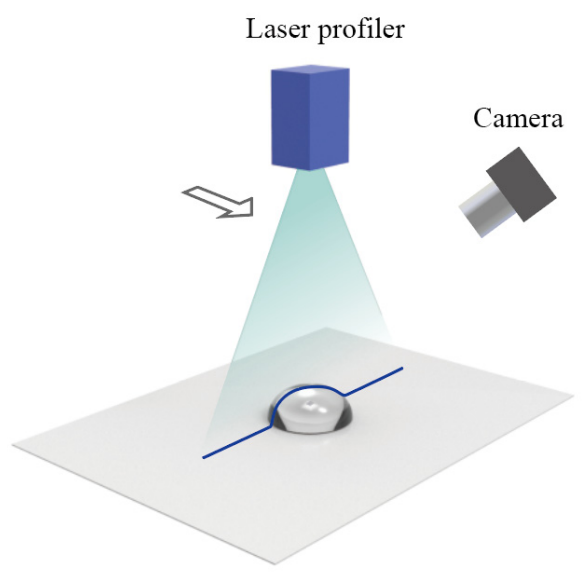

(a)

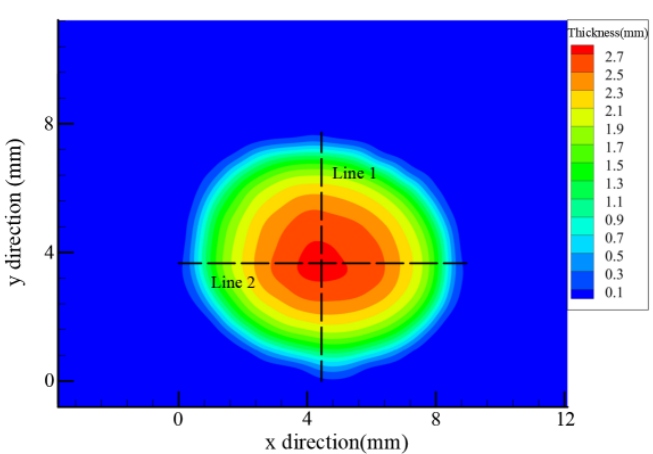

(b)

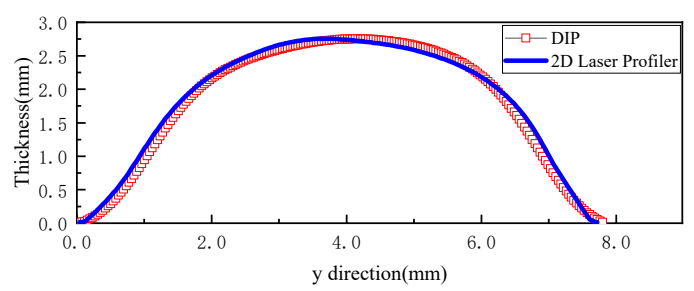

(d)

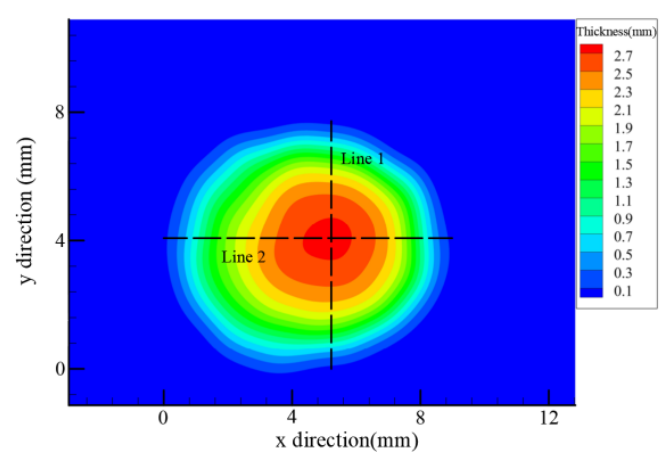

(c)

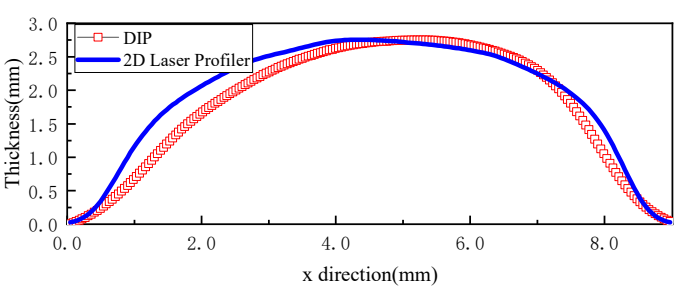

(e)

Figure 10. Measuring results of a droplet on the flat plane: (a) droplet on a flat surface; (b) result of 2D laser profiler; (c) result of DIP; (d) thickness along Line 1; (e) thickness along Line 2.

\subsection{Measurement for the Water Droplet on a Curved Surface}

Figure 11 compares the measurement results of a droplet on the cylindrical surface (identical to that introduced in Section 2.3) for the three different approaches, respectively. The droplet was located at the position with an included angle $\alpha$ of about $40^{\circ}$, as shown in Figure 11a. The measurement results of the droplet along Line 1 in Figure 11a are compared in Figure $11 \mathrm{~b}$ for the three approaches. Obviously, the measurement results acquired by different methods have different features, which are accordant with their measurement principle and limitation, respectively. Laser profiling measurement technique gives the distance along the direction of laser axis, similar to the calibration direction of 
traditional DIP technique. Unlike a flat surface, the normal direction is different at different points on a curved surface. Therefore, both laser profiler and traditional DIP can only measure the thickness along vertical direction. That was the reason for nearly the same maximum thickness (1.97 $\mathrm{mm}$ for Laser Profiler and $1.95 \mathrm{~mm}$ for traditional DIP) measured by these two methods. This observation suggests again that the traditional DIP and Laser Profiler will overestimate the real thickness of the droplets/film if they are on a curved surface. Meanwhile, because of slant arrangement of the camera (as illustrated in Figure 11a), it was not unexpected that the apex lies in the middle part of the droplet from the view of the camera.

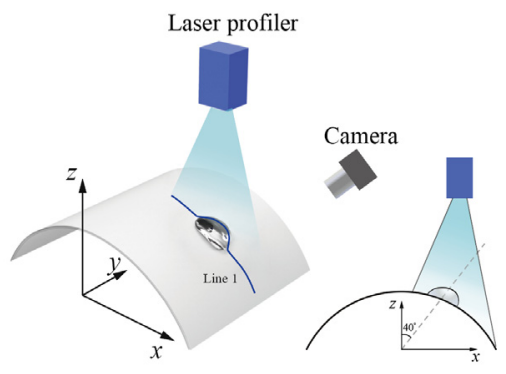

(a)

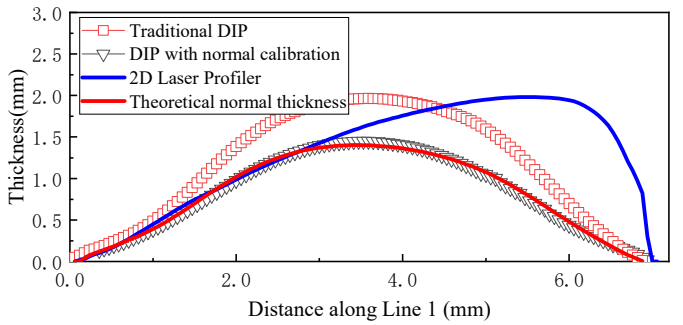

(b)

Figure 11. Measurement results of a droplet on the cylindrical surface: (a) sketch of the experimental setup; (b) thickness along Line 1.

For improved DIP with normal calibration, the measured maximum thickness was only about $1.48 \mathrm{~mm}$ - far smaller than that measured by traditional DIP method. In order to verify the accuracy of the normal calibration according to the vertical thickness acquired by traditional DIP and correction coefficients $k_{2}$ calculated by Equations (3-8), the theoretical normal thickness was obtained, shown in Figure 11b, marked with red line. The maximum error of the thickness between the present improved DIP and theoretical normal thickness was about $0.06 \mathrm{~mm}$. Therefore, the proposed DIP with normal calibration technique presents its superiority and accuracy when applied for measuring the droplets/film on a curved surface.

\section{Conclusions}

In this study, an improved digital image projection (DIP) technique with normal calibration was developed based on the traditional DIP techniques (Hu et al. [11] and Zhang et al. [12]). This improved DIP technique may be used to measure the thickness of droplets/film on curved surfaces. If the accepted error level is $5 \%$, the traditional DIP technique must be improved to eliminate systematic error when $\alpha$ is larger than $17^{\circ}$, even when $d / r$ approaches zero. Based on theoretical analyses and experimental investigations, it can be concluded that normal calibration can be realized by attaching two thin shells with given thickness to the tested curved surface, respectively. The improved DIP with normal calibration corrects systematic error caused by applying the traditional DIP to the curved surface, which can successfully provide accurate thickness in normal direction for tested objects, e.g., droplets/film.

Author Contributions: Conceptualization, Y.L.; data curation, Y.L.; software, L.Z.; supervision, H.W. and X.H.; writing-original draft, L.Z.; writing—review \& editing, H.W. and Y.L. All authors have read and agreed to the published version of the manuscript.

Funding: This research was funded by National Natural Science Foundations of China, Grant Number 11472312, U1534206.

Acknowledgments: The supports from National Natural Science Foundations of China (11472312, U1534206) are gratefully acknowledged. 
Conflicts of Interest: The authors declare no conflict of interest.

\section{References}

1. Mason, B.J. The Physics of Clouds; Clarendon: Oxford, UK, 1971.

2. Politovich, M.K. Aircraft icing caused by large supercooled droplets. J. Appl. Meteorol. 1989, 28 , 856-868. [CrossRef]

3. Hu, H.; Huang, D. Simultaneous measurements of droplet size and transient temperature within surface water droplets. AIAA J. 2009, 47, 813-820. [CrossRef]

4. Hu, H.; Jin, Z. An icing physics study by using lifetime-based molecular tagging thermometry technique. Int. J. Multiph. Flow 2010, 36, 672-681. [CrossRef]

5. Seidel, C.; Dinkler, D. Rain-wind induced vibrations-phenomenology, mechanical modelling and numerical analysis. Comput. Struct. 2006, 84, 1584-1595. [CrossRef]

6. Flamand, O. Rain-wind induced vibration of cables. J. Wind Eng. Ind. Aerodyn. 1995, 57, 353-362. [CrossRef]

7. Du, X.; Gu, M.; Chen, S. Aerodynamic characteristics of an inclined and yawed circular cylinder with artificial rivulet. J. Fluids Struct. 2013, 43, 64-82. [CrossRef]

8. Rekstad, J.; Ingebretsen, F.; Sultanovic, D.; Bjerke, B.; Grorud, C. The principles and lay-out of a new semi-open solar energy system manufactured by Solnor A/S. In Proceedings of the Third European Conference on Architecture, Florence, Italy, 17-21 May 1993.

9. Hoshiide, A.; Niidome, T.; Morooka, S.; Ishizuka, T. Study on liquid film behavior in rod bundle under adia-batic two-phase flow. In Proceedings of the 29th National Heat Transfer Sympo-sium of Japan, Osaka, Japan, 29-31 May 1992; pp. 723-724.

10. Yano, T.; Mitsutake, T.; Morooka, S.; Kimura, J. Annular two-phase flow characteristics in circular tube-spacer effect on entrainment and deposition mechanism. In Proceedings of the 2nd International Conference on Multiphase Flow, Kyoto, Japan, 3-7 April 1995.

11. Hu, H.; Wang, B.; Zhang, K.; Lohry, W.; Zhang, S. Quantification of transient behavior of wind-driven surface droplet/rivulet flows using a digital fringe projection technique. J. Vis. 2015, 18, 705-718. [CrossRef]

12. Zhang, K.; Wei, T.; Hu, H. An experimental investigation on the surface water transport process over an airfoil by using a digital image projection technique. Exp. Fluids 2015, 56, 173. [CrossRef]

(C) 2020 by the authors. Licensee MDPI, Basel, Switzerland. This article is an open access article distributed under the terms and conditions of the Creative Commons Attribution (CC BY) license (http://creativecommons.org/licenses/by/4.0/). 